\title{
5. Global Existence Theorem for Nonlinear Wave Equation in Exterior Domain
}

\author{
By Yoshihiro Shibata*) and Yoshio Tsutsumi**) \\ (Communicated by Kôsaku YosidA, M. J. A., Jan. 12, 1984)
}

The global existence of solutions for the nonlinear wave equation has been extensively studied. For the Cauchy problem Klainerman [1] has made a remarkable improvement recently. That is, he showed that if the spatial dimension is not smaller than 6 and initial data are small and smooth, then the Cauchy problem for the fully nonlinear wave equation has a unique classical global solution. On the other hand it is important to consider the initial boundary value problem for the nonlinear wave equation in an exterior domain in order to study scattering of a reflecting object for the nonlinear wave equation. In the present paper we shall announce that if the spatial dimension is not smaller than 3 and initial data are small and smooth, then we have the global unique existence theorem of classical solutions for a large class of nonlinear wave equations in exterior domains with the homogeneous Dirichlet boundary condition.

Let $\Omega$ be an unbounded domain in $\boldsymbol{R}^{n}, n \geqq 3$, with its boundary $\partial \Omega C^{\infty}$ and compact. We denote a time variable by $t$ or $x_{0}$ and a space variable by $x=\left(x_{1}, \cdots, x_{n}\right)$, respectively. We abbreviate $\partial / \partial t, \partial / \partial x_{j}$ and $\left(\partial / \partial x_{1}\right)^{\alpha_{1}} \cdots\left(\partial / \partial x_{n}\right)^{\alpha_{n}}$ to $\partial_{t}$ or $\partial_{0}, \partial_{j}$ and $\partial_{x}^{\alpha}$, respectively, where $\alpha$ is a multi-index with $|\alpha|=\alpha_{1}+\cdots+\alpha_{n}$ and $j=1, \cdots, n$. We consider the following problem :

$$
\begin{aligned}
& \Phi(u)=\square u+F(t, x, A u)=f(t, x) \quad \text { in }[0, \infty) \times \Omega, \\
& u=0 \quad \text { on }[0, \infty) \times \partial \Omega, \\
& u(0, x)=\phi_{0}(x), \quad\left(\partial_{t} u\right)(0, x)=\phi_{1}(x) \quad \text { in } \Omega,
\end{aligned}
$$

where $\partial_{t}^{2}-\Delta=\partial_{t}^{2}-\sum_{j=1}^{n} \partial_{j}^{2}$ and $A u=\left(\partial_{i} u, i=0, \cdots, n ; \partial_{j} \partial_{k} u, j, k=0, \cdots, n\right)$.

Before we state assumptions and the main theorem, we list notations. For $p$ with $1 \leqq p \leqq \infty$ we denote the standard $L^{p}$ space defined on $\Omega$ and its norm by $L^{p}(\Omega)$ and $\|\cdot\|_{p}$, respectively. For a vector valued function $h=\left(h_{1}, \cdots, h_{s}\right)$ we put

For a positive integer $N$ we put

$$
\|h\|_{p}=\sum_{j=1}^{s}\left\|h_{j}\right\|_{p}
$$

*) Department of Mathematics, University of Tsukuba. The author was supported in part by the Sakkokai Foundation.

**) Department of Pure and Applied Sciences, College of General Education, University of Tokyo. 


$$
\begin{aligned}
& \|f\|_{p, N}=\sum_{|\alpha| \leqq N}\left\|\partial_{x}^{\alpha} f\right\|_{p}, \\
& \|h\|_{p, N}=\sum_{j=1}^{s} \sum_{|\alpha| \leqq N}\left\|\partial_{x}^{\alpha} h_{j}\right\|_{p} .
\end{aligned}
$$

We set $H_{p}^{N}(\Omega)=\left\{f \in L^{p}(\Omega) ;\|f\|_{p, N}<\infty\right\} . \quad$ By $\dot{H}_{p}^{N}(\Omega)$ we denote the completion of $C_{0}^{\infty}(\Omega)$ in $H_{p}^{N}(\Omega)$. By $\mathfrak{B}^{N}(\bar{D})$ we denote the set of $C^{N}(\bar{D})$ functions having all derivatives of order $\leqq N$ bounded in $\bar{D}$, where $D$ is $\Omega,(0, \infty) \times \Omega$ or $(0, \infty) \times \Omega \times\left\{\lambda \in \boldsymbol{R}^{(n+1)(n+2)} ;|\lambda|<1\right\}$ and $\bar{D}$ is the closure of $D$. For $1 \leqq p \leqq \infty$, a nonnegative number $k$ and a nonnegative integer $N$ we put

$$
|u|_{p, k, N}=\sup _{t \geq 0}(1+t)^{k} \sum_{j+|\alpha| \leq N}\left\|\partial_{t}^{j} \partial_{x}^{\alpha} u(t, \cdot)\right\|_{p} .
$$

We make the following assumptions.

Assumptions. (1) The spatial dimension $n \geqq 3$.

(2) The nonlinear mapping $F$ is a real-valued function belonging to $\mathfrak{B}^{\infty}\left([0, \infty) \times \bar{\Omega} \times\left\{\lambda \in \boldsymbol{R}^{(n+1)(n+2)} ;|\lambda| \leqq 1\right\}\right)$.

(3)

$$
F(\lambda)= \begin{cases}O\left(|\lambda|^{2}\right) & \text { near } \lambda=0, \text { if } n \geqq 6, \\ O\left(|\lambda|^{3}\right) & \text { near } \lambda=0, \text { if } 3 \leqq n \leqq 5 .\end{cases}
$$

(4) The exterior domain $\Omega$ is "non-trapping" in the following sense: Let $G(t, x, y)$ be the Green function for the following problem

$$
\begin{aligned}
& \left(\partial_{t}^{2}-\Delta_{x}\right) G=0 \quad \text { in }(0, \infty) \times \Omega, \\
& \lim _{t \rightarrow+0} \frac{\partial^{j} G}{\partial t^{j}}= \begin{cases}0, & j=0, \\
\delta(x-y), & j=1,\end{cases} \\
& \left.G\right|_{x \in \partial \Omega}=0,
\end{aligned}
$$

where $y$ is an arbitrary point in $\Omega$ and $\Delta_{x}$ is the Laplace operator with respect to $x$. Let $a$ and $b$ be arbitrary positive constants such that $b \geqq a$ and $\partial \Omega \subset\left\{x \in \boldsymbol{R}^{n} ;|x|<a\right\}$. For any $v \in L^{2}(\Omega)$ with the support included in $\{x \in \Omega ;|x|<a\}$, we put

$$
(\boldsymbol{G} v)(t, x)=\int_{\Omega} G(t, x, y) v(y) d y .
$$

Then there exists a $T_{0}>0$ such that

$$
(\boldsymbol{G} v)(t, x) \in C^{\infty}\left(\left[T_{0}, \infty\right) \times\{x \in \bar{\Omega} ;|x| \leqq b\}\right)
$$

for any $v \in L^{2}(\Omega)$ with the support included in $\{x \in \Omega ;|x|<a\}$, where $T_{0}$ depends only on $n, a, b$ and $\Omega$.

Remark 1. It is well known that if the complement of $\Omega$ is convex, then Assumption (4) is satisfied (see, e.g., Melrose [3]).

Now we state the main theorem.

Theorem (Existence). Let $m$ be an arbitrary integer with $m \geqq 0$. Let Assumptions (1)-(4) be satisfied.

( I ) Let $n \geqq 6$. Put $\tilde{m}=2 \max (4[n / 2]+7, m+1)+4[n / 2]+8$. Then there exist positive constants $a$ and $\delta_{0}$ having the following properties : If $\phi_{0} \in \mathfrak{B}^{2 \tilde{m}+[n / 2]+3}(\bar{\Omega}), \phi_{1} \in \mathfrak{B}^{2 \tilde{m}+[n / 2]+2}(\bar{\Omega})$ and $f \in \mathfrak{B}^{2 \tilde{m}+[n / 2]+1}([0$, $\infty) \times \bar{\Omega})$ satisfy for some $\delta$ with $0<\delta \leqq \delta_{0}$ 


$$
\begin{aligned}
& \left\|\phi_{0}\right\|_{4 / 3,2 \tilde{m}}+\left\|\phi_{1}\right\|_{4 / 3,2 \tilde{m}-1}+|f|_{4 / 3,(n-1) / 4,2 \tilde{m}-2} \leqq a \delta, \\
& \left\|\phi_{0}\right\|_{4,2 \tilde{m}+2}+\left\|\phi_{1}\right\|_{4,2 \tilde{m}+1}+|f|_{4,0,2 \tilde{m}} \leqq a \delta, \\
& \left\|\phi_{0}\right\|_{\infty, 2 \tilde{m}+2}+\left\|\phi_{1}\right\|_{\infty, 2 \tilde{m}+1}+|f|_{\infty, 0,2 \tilde{m}} \leqq a \delta
\end{aligned}
$$

and the compatibility condition of order $\tilde{m}$, then Problem (M.P) has a solution $u \in C^{m+2}([0, \infty) \times \bar{\Omega})$ satisfying

$$
|\Lambda u|_{2,0, m}+|\Lambda u|_{4,(n-1) / 4, m} \leqq \delta .
$$

(II) Let $4 \leqq n \leqq 5 . \quad$ Put $\tilde{m}=2 \max (12, m+1)+13$. Then there exist positive constants $a$ and $\delta_{0}$ having the following properties: If $\phi_{0} \in \mathfrak{B}^{2 \tilde{m}+2}(\bar{\Omega}), \phi_{1} \in \mathfrak{B}^{2 \tilde{m}+1}(\bar{\Omega})$ and $f \in \mathfrak{B}^{2 \tilde{m}}([0, \infty) \times \bar{\Omega})$ satisfy for some $\delta$ with $0<\delta \leqq \delta_{0}$

$$
\begin{aligned}
& \left\|\phi_{0}\right\|_{1,2 \tilde{m}}+\left\|\phi_{1}\right\|_{1,2 \tilde{m}-1}+|f|_{1,(n-1) / 2,2 \tilde{m}-2} \leqq a \delta, \\
& \left\|\phi_{0}\right\|_{2,2 \tilde{m}+2}+\left\|\phi_{1}\right\|_{2,2 \tilde{m}+1}+|f|_{2,(n-1) / 2,2 \tilde{m}} \leqq a \delta, \\
& \left\|\phi_{0}\right\|_{\infty, 2 \tilde{m}+2}+\left\|\phi_{1}\right\|_{\infty, 2 \tilde{m}+1}+|f|_{\infty, 0,2 \tilde{m}} \leqq a \delta
\end{aligned}
$$

and the compatibility condition of order $\tilde{m}$, then problem (M.P) has a solution $u \in C^{m+2}([0, \infty) \times \bar{\Omega})$ satisfying

$$
|\Lambda u|_{2,0, m}+|\Lambda u|_{\infty,(n-1) / 2, m} \leqq \delta .
$$

(III) Let $n=3$. Let $\varepsilon$ be a positive constant with $0<\varepsilon \leqq(7 m+18)^{-1}$ and $\tilde{m}$ be an integer with $\tilde{m} \geqq(7 / \varepsilon)[3 / 2+(3 m+7) \varepsilon]+9$. Then there exist positive constants $a$ and $\delta_{0}$ having the following properties: If $\phi_{0} \in \mathfrak{B}^{2 \tilde{m}+2}(\bar{\Omega}), \phi_{1} \in \mathfrak{B}^{2 \tilde{m}+1}(\bar{\Omega})$ and $f \in \mathfrak{B}^{2 \tilde{m}}([0, \infty) \times \bar{\Omega})$ satisfy for some $\delta$ with $0<\delta \leqq \delta_{0}$

$$
\begin{aligned}
& \left\|\phi_{0}\right\|_{1,2 \tilde{m}}+\left\|\phi_{1}\right\|_{1,2 \tilde{m}-1}+|f|_{1,1+\varepsilon, 2 \tilde{m}-2} \leqq a \delta, \\
& \left\|\phi_{0}\right\|_{2,2 \tilde{m}+2}+\left\|\phi_{1}\right\|_{2,2 \tilde{m}+1}+|f|_{2,1+\varepsilon, 2 \tilde{m}} \leqq a \delta, \\
& \left\|\phi_{0}\right\|_{\infty, 2 \tilde{m}+2}+\left\|\phi_{1}\right\|_{\infty, 2 \tilde{m}+1}+|f|_{\infty, 0,2 \tilde{m}} \leqq a \delta
\end{aligned}
$$

and the compatibility condition of order $\tilde{m}$, then Problem (M.P) has a solution $u \in C^{m+2}([0, \infty) \times \bar{\Omega})$ satisfying

$$
|\Lambda u|_{2,0, m}+|\Lambda u|_{\infty, 1 / 2+\varepsilon, m} \leqq \delta .
$$

(Uniqueness). Let Assumptions (1)-(3) be satisfied. Then there exists a small constant $\delta_{1}>0$ such that if $u, v \in C^{3}([0, \infty) \times \bar{\Omega})$ are two solutions of Problem (M.P) for the same data with $|\Lambda u|_{\infty, 0,0} \leqq \delta_{1}$ and $|\Lambda v|_{\infty, 0,0} \leqq 1$, then $u=v$.

Remark 2. (1) For the compatibility condition, see Shibata [5], [6] and Shibata and Tsutsumi [7].

(2) Since the nonlinear function $F$ is defined only in $[0, \infty) \times \bar{\Omega}$ $\times\left\{\lambda \in \boldsymbol{R}^{(n+1)(n+2)} ;|\lambda| \leqq 1\right\}$, we always assume that $|\Lambda u|_{\infty, 0,0} \leqq 1$, when we consider a solution $u$ of Problem (M.P).

Our strategy of the proof of the above theorem follows Klainerman [1] and Shibata [5], [6]. Details will be published elsewhere (see Shibata and Tsutsumi [7]). 


\section{References}

[1] S. Klainerman: Global existence for nonlinear wave equations. Comm. Pure Appl. Math., 33, 43-101 (1980).

[2] S. Klainerman and G. Ponce: Global small amplitude solutions to nonlinear evolution equations. ibid., 36, 133-141 (1983).

[ 3 ] R. B. Melrose: Singularities and energy decay in acoustical scattering. Duke Math. J., 46, 43-59 (1979).

[4] J. Shatah: Global existence of small solutions to nonlinear evolution equations. J. Differential Eqs., 46, 409-425 (1982).

[5] Y. Shibata: On the global existence of classical solutions of mixed problem for some second order non-linear hyperbolic operators with dissipative term in the interior domain. Funkcial. Ekva., 25, 303-345 (1982).

[6] - : On the global existence of classical solutions of second order fully nonlinear hyperbolic equations with first order dissipation in the exterior domain. Tsukuba J. Math., 7, 1-68 (1983).

[7] Y. Shibata and Y. Tsutsumi: Global existence theorem for nonlinear wave equation in exterior domain (to appear in Lect. Notes in Numerical and Applied Analysis). Kinokuniya, Tokyo, North-Holland, Amsterdam-New York-Oxford.

[8] Y. Tsutsumi: Global solutions of the nonlinear Schrödinger equation in exterior domains (to appear in Comm. P.D.E.).

[9] - : Local energy decay of solutions to the free Schrödinger equation in exterior domains (to appear in J. Fac. Sci. Univ. Tokyo, Sect. IA, Math.). 